\title{
Editorial
}

\section{Women's perception of the benefit of breast cancer screening}

Doctors, health pamphlets from national cancer societies, and the Internet all inform women about early detection of breast cancer and the benefits of mammography screening. Thus, women should be fairly knowledgeable about screening. Yet nothing is farther from the truth, as documented by the first European-wide study [1] that included a representative sample of about 5000 women from Austria, France, Germany, Italy, Spain, Poland, the United Kingdom, and Russia (European part). Ninety-two percent of women overestimated the benefit of screening tenfold, hundredfold, two-hundredfold, or did not know; in France, Germany, and the Netherlands, the percentage rose to $98 \%$. Why are women so highly misinformed in Europe? In defence of Russian women, one might note that mammography screening is rare in Russia. Yet the Russian women made by far the most realistic estimates-likely from receiving not more but less misleading information.

What exactly are women told about the benefits of breast cancer screening? Many doctors convey an emotional message: that early detection is a moral responsibility, that women should think of their families and take care of their bodies. Pamphlets emphasize that screening can save lives but rarely provide numbers, which are more likely to be found on Web sites. If numbers about benefits are given, then most pamphlets and Web sites frame information in a format that misleads women, such as relative risk reductions [2]. Women are told that screening with mammography reduces breast cancer mortality by $20 \%$ or even $25 \%$. Elsewhere they are told that the 5-year survival rate for women who participate is $98 \%$. This is where Jane Q Public begins to get confused: Does that mean that at least 20 out of every 100 women-or even $98 \%$-who participate in screening are saved? Such confusion arises when interested parties try to exaggerate benefits and to withhold information about the risks in order to increase participation rates. The $20 \%$ and $98 \%$ figures aim at persuasion, not information. What, in contrast, would transparent information look like?

The fact box in Table 1 is such a transparent, simple, and quick tool. The two relevant questions it asks are: What are the benefits? What are the harms? The columns present the two alternatives, participating in screening or not, which makes it easy to compare the consequences of each side-by-side. Most important, the information is given in transparent numbers (e.g., out of 1000), not in terms of misleading relative risks or survival rates. Such fact boxes have been shown to foster understanding for both benefits and risks in the general public [3].

A 50-year-old woman who wants to decide whether or not to participate in screening can find the major benefits and harms in the fact box. There are two ways she can think of the benefit of screening. First, does it decrease her chances of dying from cancer,
Table 1

Breast cancer screening with mammography: per 1000 women $50+$.

\begin{tabular}{lrl}
\hline & NO screening & $\begin{array}{l}\text { Yearly screening } \\
\text { over 10 years }\end{array}$ \\
\hline Benefits? & 26 & 26 \\
$\quad$ Total cancer mortality & \\
$\quad$ Breast cancer mortality & & 4 \\
Harms? & 5 & $50-200$ \\
$\quad$ False positives with biopsies & & $2-10$ \\
$\quad$ Unnecessary treatments & & \\
$\quad$ (e.g., lumpectomy) & &
\end{tabular}

a Calculated from [4], analysis 01.07.

b Based on [4], analysis 01.06, and [5].

say within the next 10 years? Second, does it decrease her chances of dying from breast cancer? The fact box indicates that according to the best evidence available, the answer to the first question is "no." The answer to the second question is "yes"; screening reduces the number of women who die from breast cancer from 5 to 4 in 1000 , that is, by 1 in 1000 . The fact box also lists the potential harms, which are reported for the same reference class of 1000 women. About 50-200 women without breast cancer will undergo unnecessary biopsies and anxieties due to false positives, and 2-10 women with non-progressive or slow-growing breast cancer will undergo unnecessary treatments such as lumpectomies or mastectomies. For these women, screening provides no benefits but negatively affects their quality of life. A third potential harm, getting radiation-induced breast cancer from the mammography, is not included because only rough indirect estimates (1-5 in 10,000) exist. The simple fact box enables women to come up with informed decisions on an individual basis, according to their priorities.

Fact boxes of this type allow patients to see through misleading information. Consider the 20\% risk reduction: from 5 to 4 in 1000 , equivalent to 1 in 1000 . This $20 \%$ figure may be the reason why $27 \%$ of women in the U.K. believe that mammography screening saves 200 out of 1000 women from dying from breast cancer, whereas only $7 \%$ of Russian women think so [1]. The $98 \%$ survival rate is another deceptive tool, given that changes in 5-year survival rates have no reliable relationship to changes in mortality. Yet to the targeted women, higher survival mistakenly implies lower mortality. One reason for this confusion is the so-called lead-time bias: Screening leads to early detection, which in turn inflates the 5 -year survival rate, because survival is measured from the time of detection. A second reason is overdiagnosis, that is, screening detects non-progressive cancers that by definition do not lead to mortality, yet their number increases the survival rate [2]. 
The fact that $92 \%$ of European women overestimate the benefits of mammography tenfold, hundredfold, two-hundredfold, or do not know, should give everyone pause for thought. The blame should not be on the women, who are only the last element in a long production line that actively creates and maintains health illiteracy. Physicians, health pamplets, and journalists contribute, often unawares. For instance, one-third of hundreds of gynecologists I have trained in their continuing education did not even know the benefit of mammography screening but mistakenly believed that a $25 \%$ risk reduction meant that 25 or 250 fewer women out of 1000 die from breast cancer [2]. In the European study, we discovered that women who frequently consulted general practitioners or health pamphlets overestimated the benefits even more than those who did not.

Misleading women, whether intentionally or unintentionally, about the benefit of mammography screening is a serious issue. All of those in the business of informing women about screening should recall that medical systems are for patients, not the other way around.

\section{Provenance and peer review}

Commissioned, not externally peer reviewed.

\section{Competing interest}

No competing interests.

\section{References}

[1] Gigerenzer G, Mata J, Frank R. Public knowledge of benefits of breast and prostate cancer screening in Europe. JNCI 2009;101:1216-20.

[2] Gigerenzer G, Gaissmaier W, Kurz-Milcke E, Schwartz LM, Woloshin SW. Helping doctors and patients make sense of health statistics. Psychol Sci Public Interest 2007;8:53-96.

[3] Schwarz LM, Woloshin S, Welch H. The drug fact box: providing consumers with simple tabular data on drug benefit and harm. Med Dec Making 2007;27:655.

[4] Gøtzsche PC, Nielsen M. Cochrane Database Syst Rev 2006, 4. Article CD001877. DOI: 001810.001002/14651858.CD14001877.pub14651852.

[5] Woloshin S, Schwarz LM. JNCI 2009;101:17.

Gerd Gigerenzer* Max Planck Institute for Human Development, Lentzeallee 94, 14195 Berlin, Germany

*Tel.: +493082604460 E-mail address: gigerenzer@mpib-berlin.mpg.de

8 June 2010 